Article

\title{
Sensitivity Analysis of 3D Individual Tree Detection from LiDAR Point Clouds of Temperate Forests
}

\author{
Wei Yao $^{1, *}$, Jan Krull ${ }^{1}$, Peter Krzystek ${ }^{1}$ and Marco Heurich ${ }^{2}$
}

1 Department of Geoinformatics, University of Applied Sciences-Munich, Munich 80333, Germany; E-Mails: jan.krull@hm.edu (J.K.); krzystek@hm.edu (P.K.)

2 Bavarian Forest National Park, Grafenau 94481, Germany;

E-Mail: marco.heurich@npv-bw.bayern.de

* Author to whom correspondence should be addressed; E-Mail: yao@hm.edu;

Tel.: +49-89-1265-2632; Fax: +49-89-1265-2698.

Received: 4 March 2014; in revised form: 8 April 2014 / Accepted: 14 May 2014 /

Published: 28 May 2014

\begin{abstract}
Light detection and ranging (LiDAR) sampling or full-area coverage is deemed as favorable means to achieve timely and robust characterizations of forests. Recently, a 3D segmentation approach was developed for extracting single trees from LiDAR data. However, key parameters for modules used in the strategy had to be empirically determined. This paper highlights a comprehensive study for the sensitivity analysis of 3D single tree detection from airborne LiDAR data. By varying key parameters, their influences on results are to be quantified. The aim of the study is to enlighten the optimal combination of parameter values towards new applications. For the experiment, a number of sample plots from two temperate forest sites in Europe were selected. LiDAR data with a point density of $25 \mathrm{pts} / \mathrm{m}^{2}$ over the first site in the Bavarian forest national park were captured with under both leaf-on and leaf-off conditions. Moreover, a Riegl scanner was used to acquire data over the Austrian Alps forest with four-fold point densities of $5 \mathrm{pts} / \mathrm{m}^{2}$, $10 \mathrm{pts} / \mathrm{m}^{2}, 15 \mathrm{pts} / \mathrm{m}^{2}$ and $20 \mathrm{pts} / \mathrm{m}^{2}$, respectively, under leaf-off conditions. The study results proved the robustness and efficiency of the 3D segmentation approach. Point densities larger than $10 \mathrm{pts} / \mathrm{m}^{2}$ did not seem to significantly contribute to the improvement in the performance of $3 \mathrm{D}$ tree detection. The performance of the approach can be further examined and improved by optimizing the parameter settings with respect to different data properties and forest structures.
\end{abstract}


Keywords: tree detection; segmentation; temperate forests; sensitivity analysis; airborne LiDAR

\section{Introduction}

Airborne laser scanning (ALS) or Light detection and ranging (LiDAR) has been widely used in mapping the Earth's surface including urban and forested areas in 3D. Recent advances in LiDAR technology have demonstrated that new full waveform scanners can provide a higher spatial point density especially along the vertical dimension and additional information about the reflectional characteristics and vertical structure of trees [1-5]. As LiDAR instrument technology continues to improve and acquisition costs decrease, the wall-to-wall characterization of large forested areas will become more common. Therefore, LiDAR sampling or full-area coverage is deemed as favorable means to achieve timely and robust large-area characterizations of vertically and horizontal distributed forest structures.

Two main strategies on the use of LiDAR data in forestry are reported: the area-based method (ABA) and the individual tree method (ITD). In the ABA, ALS point cloud data are aggregated at the inventory plot level by describing them based on the height distribution and canopy density metrics [6-8], such as echo ratio, height percentiles and canopy cover percentiles. These metrics can be imported into regression models as independent variables. In this way, the response is an aggregation of single tree measurements on sample plots and could be as in the case of mean tree height, stem volume, biomass or forest fuel parameters [9-13].

In the ITD approach, either the canopy height model (CHM) [14,15] or the ALS raw data point clouds $[3,16,17]$ are used to be segmented into single tree objects. Properties at single tree level can subsequently be estimated using the tree segments, such as tree height, crown diameter, tree species, crown base height and stem volume. The ITD method is more intuitive than ABA since the derived parameters refer to the single tree, which is the smallest unit in forest management. Novel methods for ITD approach tackle conceptually the segmentation problem with a 3D approach instead of using only the CHM. In combination with full waveform data Reitberger et al. [16] successfully demonstrated that the overall detection rate of single trees could be significantly improved, especially in heterogeneous forest types. The improvement happened mostly to the lower forest layers with $20 \%$. The fusion of 3D techniques with full waveform data pushed the single tree approach to a new level of completeness. Meanwhile, the estimation of tree shape parameters benefits from 3D tree segments. Moreover, the tree reflectional characteristics gain more insights into tree structure which are significant for tree species classification and growth condition identification. The physical reflectivity property of trees could even be enhanced and better described if echo amplitude of full waveform ALS data were normalized with respect to different incidence angles [18]. Therefore, unbiased species-specific models can be used to accurately determine forest biophysical parameters even at single tree level $[3,19]$.

Recently, an international comparison study on ITD from ALS data [20] has indicated that the extraction method is the main factor impacting achieved accuracy rather than laser point density. So 
far, there is lack of a comprehensive sensitivity analysis of detecting 3D individual trees in heterogeneous forest areas using LiDAR data. A fully automated method for tree detection is always desirable; a sensitivity analysis is performed to characterize and quantify the uncertainty sources of the algorithm and help optimize the selection of control parameters towards the best performance under different forest conditions. Therefore, a detailed understanding of the impact of the varying key control parameters on the performance of tree detection is required. In the former work of Reitberger et al. [16], the smoothing factor and segmentation threshold were already investigated in a preliminary configuration with a limited point density and value domain. In the past several years, a few authors have been dealing with similar topics. In [21,22], an uncertainty analysis is performed to estimate forest carbon stock and biomass from satellite imagery and LiDAR data. The dominate sources of uncertainty are the variation of input sample plot data and data saturation problems related to optical sensors. Monnet et al. [23] conducted a sensitivity analysis of a treetop detection algorithm in the French Alps by automated evaluation of detection performance for several parameter combinations. The optimization of parameters may depend on both the laser data, mainly point density, and on the forest structure and species. Palleja et al. [24] investigated the sensitivity of the tree volume estimates relative to different error sources in the spatial trajectory of a mobile terrestrial LiDAR and found out that the volume estimation was very sensitive to both errors in the determination of the distance from the LiDAR to the center of the trees and in the determination of the orientation angle of LiDAR. In [25], fuel model maps obtained based on QuickBird image and LiDAR-derived variables were used to perform a sensitivity analysis of fire behavior modeling. The study showed that the accuracy of fuel mapping could be improved by at least $13 \%$. The objectives of this work are (i) to examine the influence of the uncertainty of control parameters on the performance of tree detection in mixed strand forests from airborne full-waveform LiDAR; (ii) to exploit the feasibility of optimizing parameter settings; and (iii) to perform a sensitivity analysis of 3D tree detection from airborne LiDAR data.

The paper is divided into five sections. Section 2 focuses on the methodology of 3D segmentation of single trees and introduces the key control parameters that undergo the sensitivity analysis. Section 3 presents the experimental results obtained from full-waveform LiDAR data of four-fold point densities in the Austrian floodplain forest. Finally, results are discussed and concluded in the last two sections.

\section{Methodology}

\subsection{Single Tree Detection}

The hybrid approach for single tree detection presented in former work [16] consists of three steps: waveform decomposition, watershed transformation with stem detection and normalized cut segmentation within merged watershed segments.

A single waveform is decomposed by fitting mixed Gaussian pulse models to the backscattered waveform which contains $N_{R}$ reflections. The vector $X_{i}^{T}=\left(x_{i}, y_{i}, z_{i}, W_{i}, I_{i}\right)$ is provided for each reflection $i$ with the 3D coordinates of the echo. Additionally, the points $X_{i}$ are given by the width $W_{i}$ and the intensity $I_{i}$ of the echo pulse [5]. The LiDAR data are corrected by referencing $W_{i}$ and $I_{i}$ to the 
pulse width and the intensity of the emitted Gaussian pulse and by correcting the intensity with respect to the run length $S_{i}$ (distance between laser sensor and target) of the laser beam and a nominal distance.

The coarse detection of single trees is achieved from the CHM using a watershed transformation. The CHM is derived by subdividing the Region of Interest (ROI) into a grid with having a cell spacing of $c p$ and $N_{C}$ cells. Within each grid cell, the highest 3D point is selected and adapted with respect to the ground level $z_{j}^{\text {ground }}$. Based on bilinear interpolation, the ground level $z_{j}^{\text {ground }}$ is estimated from a given digital terrain model (DTM) with a grid size of $1 \mathrm{~m}$ and an absolute accuracy of $25 \mathrm{~cm}$ [11]. In the next step, all the highest $3 \mathrm{D}$ points $X_{j}^{T}=\left(x_{j}, y_{j}, z_{j}^{C H M}\right)\left(j=1, \ldots, N_{C}\right.$ ) of all $N_{C}$ cells are robustly interpolated in a grid that has $N_{X}$ and $N_{Y}$ grid lines and a grid size $g_{w}$. For this purpose an algorithm "gridfit" [26] is adopted which smoothens the surface by maintaining the surface gradients as small as possible. The trade-off between interpolation and regularization is determined by the adjustable smoothing factor $\lambda$. Both steps are carried out simultaneously in a least squares adjustment. The watershed segments derived on this $\mathrm{CHM}$ act as candidate regions where single trees are contained.

Within every watershed segments the 3D segmentation technique using normalized cuts [27] is used to detect point clouds associated with single trees (Figure 1). This segmentation uses the positions $\left(x_{i}, y_{i}, z_{i}\right)$ of the laser reflections and the pulse width $W_{i}$ and the intensity $I_{i}$ obtained from the waveform decomposition. Additionally, stem positions or local maxima derived from the watershed segmentation of CHM serve as prior knowledge. The normalized cut segmentation applied to the voxel structure of a watershed segment is based on a graph $G$ describing the adjacent topology between each voxel (Figure 1). A pair of two disjoint Segments $A$ and $B$ of the graph is found by minimizing the cost function:

$$
\operatorname{NCut}(A, B)=\frac{\operatorname{Cut}(A, B)}{\operatorname{Assoc}(A, V)}+\frac{\operatorname{Cut}(A, B)}{\operatorname{Assoc}(B, V)}
$$

with $\operatorname{Cut}(A, B)=\sum_{i \varepsilon A, j \varepsilon B} w_{i j}$ as the total sum of weights between the Segments $A$ and $B$ and $\operatorname{Assoc}(A, V)=\sum_{i \varepsilon A, j \varepsilon V} w_{i j}$ as the sum of the weights of all edges ending in Segment $A$. The weights $w_{i j}$ specify the similarity between the voxels and are a function of the LiDAR point distribution and various features, which are defined in Equation (2). The segmentation is realized by maximizing the similarity within the segment voxels and minimizing the similarity between Segments $A$ and $B$. The graph $G$ is recursively bi-partitioned until a stop criterion (given the normalized cut value $N C u t_{\text {Thres}}$ ) is met; the spatial distribution of the LiDAR points mainly influences the weighting function. The features derived from the LiDAR points attributes of $W_{i}$ and $I_{i}$ also support the segmentation result. 
Figure 1. (a) Subdivision of the region of interest (ROI) into a voxel structure [16];

(b) Local maximum in canopy height model (CHM).

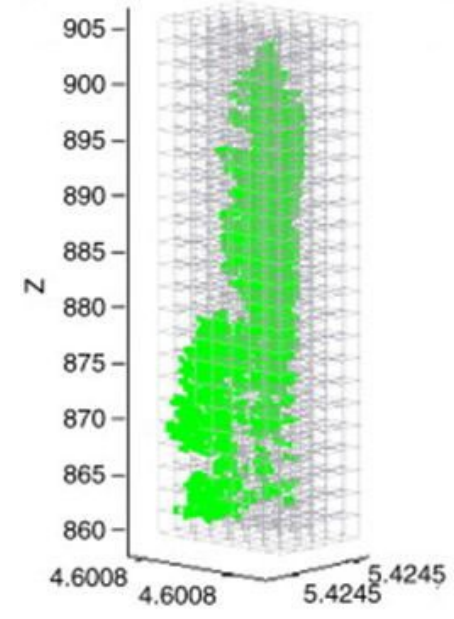

(a)

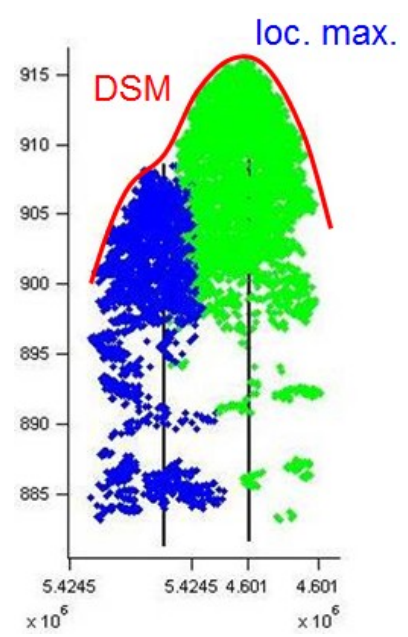

(b)

One of the key factors is to set up the weighting matrix $W$ with the similarity reflecting the likelihood that two voxels belong to one tree. The similarity measure between each pair of voxels is the multiplicative exponential model:

$$
w(i, j)=\left\{\begin{array}{c}
e^{-X(i, j)} \times e^{-Z(i, j)} \times e^{-F(i, j)} \times e^{-G(i, j)} \text { if }\left(D_{i j}^{X Y}<r_{x y}\right) \\
0 \text { otherwise }
\end{array}\right.
$$

with $X(i, j)=\left(\frac{D_{i j}^{X Y}}{\sigma_{x y}}\right)^{2}, Z(i, j)=\left(\frac{D_{i j}^{Z}}{\sigma_{z}}\right)^{2}, F(i, j)=\left(\frac{\left|f_{i}-f_{j}\right|}{\sigma_{f}}\right)^{2}, G(i, j)=\left(\frac{G_{i j}^{\max }}{\sigma_{G}}\right)^{2}$ that computes the similarities $w_{i j}$ between two voxels $i$ and $j$ within a cylinder of radius $r_{x y}$ around the voxel $i$. The components $X(I, j)$ and $Z(i, j)$ weight quadratic horizontal and vertical Euclidian distances between voxels. They are weighted separately to take into account information about the typical tree shape. The component $F(i, j)$ describes the quadratic Euclidian distance between two feature vectors (mean pulse intensity $I_{\text {mean }}$ and pulse width $W_{\text {mean }}$ ) derived from the data points (=reflections) in the voxels. The fraction $G(I, j)$ models the dependency of two voxels $i$ and $j$ of the local maxima by the maximal horizontal distance $G_{i j}^{\max }$. Hence, it is modeled that voxels nearby belong most probably belong to one tree.

\subsection{Key Control Parameters for Sensitivity Analysis}

Several key control parameters for 3D tree extraction algorithm from airborne LiDAR data are selected here to perform the sensitivity analysis.

\subsubsection{Threshold for Normalized Cut Value $N C u t_{\text {Thres }}$}

The minimization of $\operatorname{NCut}(A, B)$ is solved by the corresponding generalized eigenvalue problem $(D-W) \times y=\lambda \times D y$, where $n \times n$ weighting matrix $W$ represents weights $w_{i j}$ between all $n$ nodes of the graph $G$. The minimum solution $y_{1}$ corresponds to the second smallest eigenvalue. Since $y_{1}$ is 
real-valued and may only have two distinct indicator values $(+1,-1)$ we need to binarize it by introducing a threshold into the histogram of $y_{1}$. Using simply $t$ value 0 or median $\left(y_{1}\right)$ leads to proper results. The results can be improved by testing several possible values with respect to the resulting value of $N C u t$ and choosing the value causing the smallest $N C u t$ value. Thus, the graph $G$ is subdivided into two disjoint segments $G_{1}$ and $G_{2}$. After that, the abovementioned steps of solving the eigenvalue problem and binarization of $y_{1}$ are to be performed iteratively on the sub-graphs $G_{1}$ and $G_{2}$ until the value for $N C u t$ reaches or exceeds the threshold $N C u t_{\text {Thres. }}$ Figure 2 shows an illustrative example of recursive normalized cut for a five-tree case study. One can observe that the way in which the tree segmentation is performed is dependent on the selection of the threshold $N C u t_{\text {Thres. }}$ Therefore, the sensitivity of tree detection performance with respect to the variation of $N C u t_{\text {Thres }}$ value needs to be examined in detail.

Figure 2. Recursive normalized cut for a five-tree example by progressively increasing the NCut ${ }_{\text {Thres }}$ value (green line) [28].
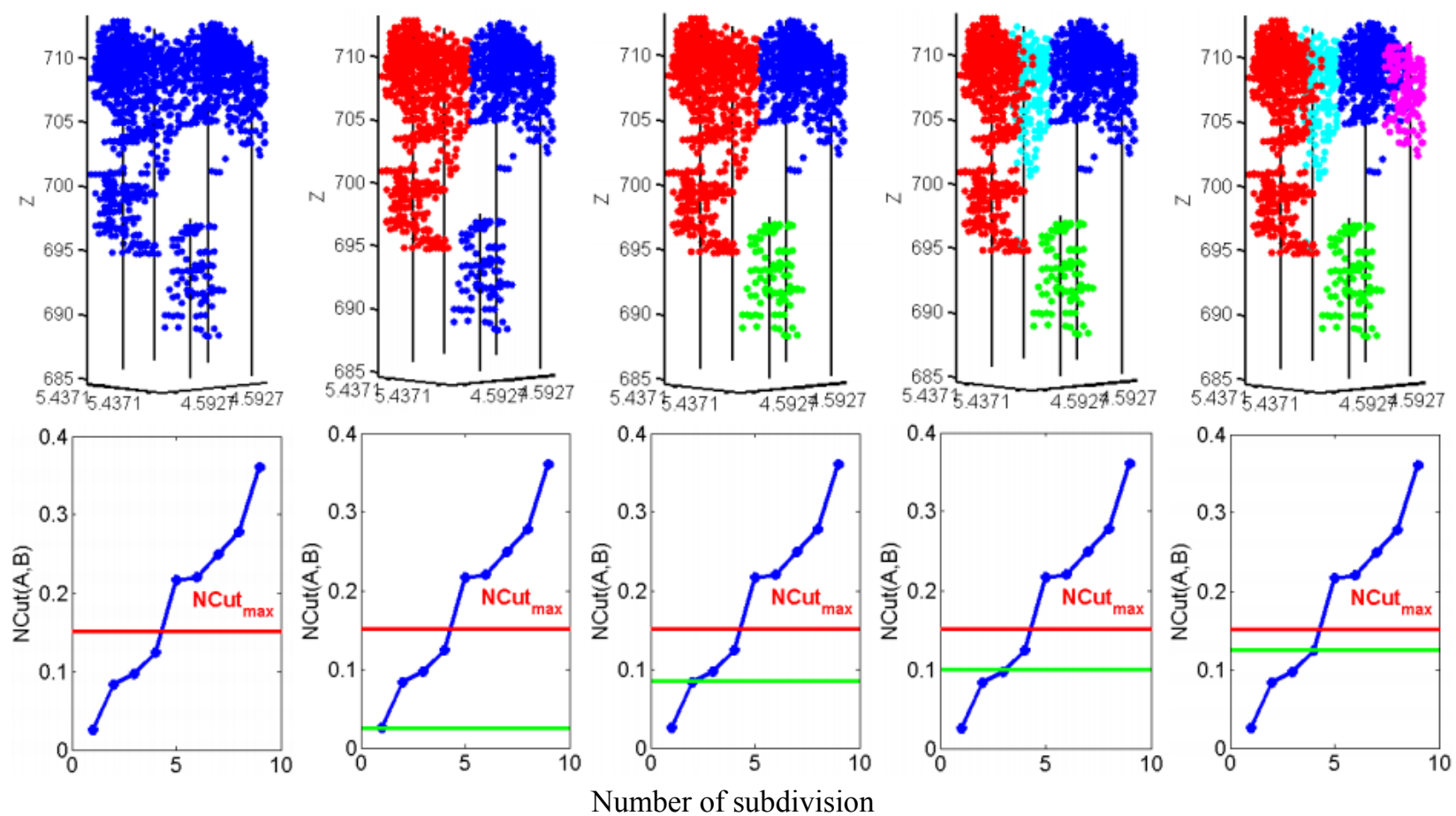

2.2.2. Voxel Size $V_{\text {size }}$

To enable a 3D tree segmentation in LiDAR point clouds based on normalized cuts the ROI delineated by the watershed segmentation is subdivided into a voxel structure with a voxel spacing of $V_{\text {size }}$ and $N_{v}=N_{v}^{x} \times N_{v}^{y} \times N_{v}^{z}$ voxels (Figure 1a). Within each voxel of the size $V_{\text {size }}$ we collect $N$ reflections $X_{i}^{T}=\left(x_{i}, y_{i}, z_{i}, W_{i}, I_{i}\right)(i=1 \ldots N)$, where only voxels comprising at least one reflection are used in the segmentation. The voxel structure is represented as a region adjacency graph $G=\{V, E\}$ with $V$ as the voxels representing the nodes and $E$ as the edges formed between every pair of nodes. The similarity between two nodes $\{i, j\} \in V$ is described by the weights $w_{i j}$ which are computed from features associated with the voxels. Basically, the similarity between voxels decreases 
with increasing distance between two voxels and drops down to zero beyond the threshold $r_{X Y}$ for the mutual distance in order to keep the graph $G$ at a reasonable size for computation.

\subsubsection{Smoothing Factor $\lambda$ for CHM Generation.}

The watershed transformation is performed at the first step to segment the CHM and delineate 2D crowns of dominator trees. The watershed segments and local maxima can be viewed as candidate areas and tree positions for 3D tree segmentation under the CHM. For this purpose the "gridfit" method is adopted to generate CHM from point clouds, which smoothens the surface by keeping the surface gradients as small as possible. The balance between interpolation and regularization is determined by the adjustable smoothing factor $\lambda$. Both steps are carried out simultaneously in a least squares adjustment scheme. The result is a smoothed CHM having equally spaced posts to which the watershed segmentation is applied. Note that the factor $\lambda$ controls both the smoothing and regularization in the case of ill posed situations. The local maxima of the watershed segments define possible tree positions. This segmentation procedure is mainly controlled by the parameter $\lambda$ for the CHM construction, while the watershed algorithm itself does not include any parameters.

Moreover, the parameter $\lambda$ affects the normalized cut segmentation, since the weighting matrix $W$ in Equation.(2) describing the similarity measures between each two voxels incorporated the term $e^{-G(i, j)}$ Note that a certain smoothing of the CHM is necessary in order to avoid an oversegmentation with higher false alarms. On the other hand, neighboring trees often merge and thereby and form a tree group instead of single trees. Furthermore, the CHM should not be over-smoothed either, since the number of local maxima is directly related to the number of detected trees and fewer local maxima lead to a weaker evidence of the presence of single trees within the watershed segments. It is expected to see which kind of impact on the performance of $3 \mathrm{D}$ tree detection the smoothing factor could have.

\section{Experiment}

\subsection{Material}

\subsubsection{Test Site I}

One of the experiments was conducted in the Bavarian Forest National Park (49 $3^{\prime} 19^{\prime \prime}$ N, $13^{\circ} 12^{\prime} 9^{\prime \prime} \mathrm{E}$ ), which is located in South-Eastern Germany along the border to the Czech Republic (Figures 5 and 6). There are two major test sites of size between 591 ha and 954 ha in size containing sub alpine spruce forest, mixed mountain forest and alluvial spruce forest as the three major forest types. Furthermore, the trees are subdivided into three layers with respect to the top height $h_{\text {top }}$ of the plot, where $h_{\text {top }}$ is defined as the average height of the 100 highest trees per ha. The lower layer contains all trees below $50 \%$ of $h_{\text {top }}$, the intermediate layer refers to all trees between $50 \%$ and $80 \%$ of $h_{\text {top }}$, and finally, the upper layer contains the rest of the trees. Table 1 summarizes the characteristics of the individual sample plots in Test Site I. 
Table 1. Characteristics of sample plots in Test Site I; N stands for the number of trees.

\begin{tabular}{cccccccc}
\hline Plot & Size (ha) & Age (a) & Trees/ha & Deciduous (\%) & $\begin{array}{c}\text { N lower } \\
\text { layer }\end{array}$ & $\begin{array}{c}\text { N interm. } \\
\text { layer }\end{array}$ & $\begin{array}{c}\text { N upper } \\
\text { layer }\end{array}$ \\
\hline 21 & 0.20 & 160 & 500 & 66 & 37 & 14 & 48 \\
22 & 0.20 & 160 & 540 & 79 & 19 & 60 & 29 \\
55 & 0.15 & 240 & 830 & 5 & 77 & 21 & 20 \\
56 & 0.23 & 170 & 340 & 10 & 31 & 19 & 27 \\
57 & 0.10 & 100 & 450 & 0 & 0 & 4 & 41 \\
58 & 0.10 & 85 & 440 & 14 & 10 & 4 & 30 \\
59 & 0.10 & 40 & 2150 & 1 & 76 & 85 & 54 \\
60 & 0.10 & 110 & 380 & 100 & 8 & 22 & 27 \\
64 & 0.12 & 100 & 430 & 87 & 13 & 4 & 35 \\
65 & 0.12 & 100 & 810 & 96 & 53 & 26 & 35 \\
74 & 0.30 & 85 & 700 & 29 & 11 & 33 & 165 \\
81 & 0.30 & 70 & 610 & 100 & 29 & 59 & 96 \\
91 & 0.36 & 110 & 260 & 75 & 31 & 11 & 54 \\
92 & 0.25 & 110 & 170 & 100 & 13 & 3 & 27 \\
93 & 0.28 & 110 & 240 & 66 & 7 & 2 & 59 \\
94 & 0.29 & 110 & 250 & 97 & 15 & 4 & 54 \\
95 & 0.25 & 110 & 240 & 10 & 6 & 0 & 53 \\
96 & 0.30 & 110 & 200 & 86 & 30 & 3 & 26 \\
\hline
\end{tabular}

Full-waveform LiDAR data of several ALS campaigns are available for the test sites, which have been collected by Milan Flug GmbH with the Riegl LMS-Q560 system. Table 2 contains details about the point density, leaf-on and leaf-off conditions during the flights and the footprint size. The term point density refers to the nominal value influenced by the pulse repetition rate (PRF), flying altitude, flying speed and strip overlap. Naturally, the reference data have been updated for the individual flying dates. The calibration of the Riegl full waveform system was determined from special calibration flights performed over an airfield in order to make the pulse intensity consistent with respect to the varied flight altitude. Several tracks were flown at different flying heights $(200 \mathrm{~m}$ and $400 \mathrm{~m})$ along and across the airfield. The mean intensity $I_{i}$, corrected with respect to the emitted intensity $I^{e}$, and the mean distance from the LiDAR to the target $s_{i}$ were calculated in four homogeneous areas $\left(122 \mathrm{~m}^{2}-133 \mathrm{~m}^{2}\right)$ for each track $i$. In order to reduce the impact of the scan angle we only used measurements whose scan angles were smaller than 10 degrees. The best coefficient $k$ was estimated from all possible observation equations (3):

$$
I_{i} \times S_{i}^{k}=I_{j} \times S_{j}^{k}
$$

which can be formulated for two tracks $i$ and $j$ flown at different heights. Table 2 also shows the results obtained for the two flights of leaf-off and on. However, no detailed information from the hardware supplier is available on that subject. The calibrated values for $k$ were used in the following analysis. 
Table 2. Configurations for airborne light detection and ranging (LiDAR) campaigns for Test Site I.

\begin{tabular}{lll}
\hline \multicolumn{1}{c}{ Time of flight } & \multicolumn{1}{c}{ May 2006 } & \multicolumn{1}{c}{ May 2007 } \\
\hline Data set & I & II \\
Foliage & Leaf-off & Leaf-on \\
Scanner & Riegl LMS-Q560 & Riegl LMS-Q560 \\
Pts $/ \mathrm{m}^{2}$ & 25 & 25 \\
Above ground level (AGL) (m) & 400 & 400 \\
Beam divergence (mrad) & $\leq 0.5$ & $\leq 0.5$ \\
Calibration parameter k & 1.902 & 1.736 \\
\hline
\end{tabular}

\subsubsection{Test Site II}

The second experiment was conducted in the Austrian floodplain forest, which is located in western Austria along the border to the Germany $\left(47^{\circ} 58^{\prime} 19^{\prime \prime} \mathrm{N}, 12^{\circ} 55^{\prime} 9^{\prime \prime} \mathrm{E}\right)$. Seven sample plots with an area size between $200 \mathrm{~m}^{2}$ and $500 \mathrm{~m}^{2}$ were selected. The forest comprises alluvial forest, spruce monocropping, and deciduous mixed forest with partial pinewood. Practically all age classes are found. The plots comprise forest in the early and the late pole phase. Table 3 summarizes the characteristics of the individual sample plots in Test Site II.

Table 3. Characteristics of sample plots in Test Site II; N stands for the number of trees.

\begin{tabular}{|c|c|c|c|c|c|c|c|}
\hline Plot & Size (ha) & Altitude (m) & Trees/ha & Deciduous (\%) & N lower layer & $\mathbf{N}$ intern layer & $\mathbf{N}$ upper layer \\
\hline 1 & 0.05 & 441 & 448 & 0 & 0 & 0 & 12 \\
\hline 2 & 0.04 & 441 & 483 & 0 & 0 & 0 & 9 \\
\hline 3 & 0.05 & 441 & 417 & 100 & 0 & 2 & 11 \\
\hline 4 & 0.04 & 441 & 349 & 100 & 0 & 1 & 7 \\
\hline 5 & 0.05 & 441 & 490 & 0 & 0 & 0 & 13 \\
\hline 6 & 0.04 & 440 & 261 & 100 & 0 & 2 & 2 \\
\hline 7 & 0.04 & 440 & 202 & 100 & 0 & 1 & 4 \\
\hline 8 & 0.03 & 441 & 560 & 75 & 0 & 6 & 2 \\
\hline 9 & 0.05 & 441 & 453 & 0 & 0 & 0 & 14 \\
\hline 10 & 0.05 & 440 & 441 & 0 & 0 & 0 & 13 \\
\hline 11 & 0.04 & 440 & 272 & 100 & 0 & 0 & 5 \\
\hline 12 & 0.05 & 439 & 196 & 100 & 0 & 0 & 6 \\
\hline 13 & 0.05 & 439 & 487 & 0 & 0 & 0 & 14 \\
\hline 14 & 0.05 & 439 & 490 & 0 & 0 & 0 & 13 \\
\hline 15 & 0.06 & 439 & 679 & 0 & 0 & 0 & 23 \\
\hline 16 & 0.04 & 440 & 371 & 100 & 0 & 2 & 7 \\
\hline 17 & 0.05 & 439 & 698 & 0 & 0 & 0 & 18 \\
\hline 18 & 0.05 & 482 & 576 & 0 & 0 & 0 & 16 \\
\hline 19 & 0.05 & 483 & 633 & 12 & 0 & 0 & 6 \\
\hline 20 & 0.05 & 468 & 631 & 94 & 9 & 4 & 3 \\
\hline 21 & 0.05 & 464 & 405 & 90 & 4 & 2 & 3 \\
\hline 22 & 0.05 & 464 & 690 & 0 & 0 & 0 & 17 \\
\hline 23 & 0.04 & 448 & 330 & 0 & 0 & 0 & 5 \\
\hline 24 & 0.05 & 447 & 471 & 83 & 1 & 2 & 6 \\
\hline 25 & 0.05 & 456 & 692 & 0 & 0 & 0 & 19 \\
\hline 26 & 0.04 & 442 & 340 & 0 & 0 & 2 & 6 \\
\hline 27 & 0.04 & 442 & 411 & 0 & 0 & 1 & 7 \\
\hline
\end{tabular}


Full waveform data were collected by Forest Mapping Management GmbH in Salzburg with the Rieg1 LMS-Q680i in March 2011 after snowmelt but prior to foliation with a four-fold average point density of 5-20 points $/ \mathrm{m}^{2}$ (Table 4). The laser pulse repetition rate was $240 \mathrm{kHz}$, the pulse width at half maximum reached $4 \mathrm{~ns}$ and the laser wavelength was $1550 \mathrm{~nm}$. The flying altitude of $700 \mathrm{~m}$ resulted in a footprint size of ca. $30 \mathrm{~cm}$.

Table 4. Configurations of the airborne LiDAR campaign in Test Site II.

\begin{tabular}{ll}
\hline \multicolumn{1}{c}{ Time of flight } & March 2011 \\
\hline Foliage & Leaf-off \\
Scanner & Riegl LMS-Q680i \\
Point density: Pts $/ \mathrm{m}^{2}$ & $5,10,15,20$ \\
AGL (m) & 700 \\
Beam divergence (mrad) & $<=0.5$ \\
Scan angle & $0^{\circ}-22.5^{\circ}$ \\
\hline
\end{tabular}

Unfortunately, there were no special calibration flights performed in this data acquisition campaign to determine the calibration of the Riegl full waveform laser system. Therefore, for this test site the mean intensity $I_{i}$ was calculated by the correction only with respect to the emitted intensity $I_{e}$, and the mean run length $\mathrm{s}_{i}$ for each track, simply assuming simply the coefficient $k=2$ for all flights.

\subsection{Field Data and Evaluation}

\subsubsection{Test Site I}

At the Test Site I, we selected 18 sample plots with an area size between $1000 \mathrm{~m}^{2}$ and $3600 \mathrm{~m}^{2}$ in two test sites $\mathrm{E}$ and $\mathrm{C}$. Reference data for all trees with $\mathrm{DBH}$ larger than $7 \mathrm{~cm}$ were collected for 688 Norway spruces (Picea abies), 812 European beeches (Fagus sylvatica), 70 fir trees (Abies alba), 71 Sycamore maples (Acer pseudoplatanus), 21 Norway maples (Acer platanoides) and two lime trees (Tilia Europaea). Tree parameters like the DBH, total tree height, stem position and tree species were measured and determined by GPS, tachometry and the "Vertex III" system. Naturally, the reference data were updated for the individual dates of the LiDAR data. A DTM with a grid size of $1 \mathrm{~m}$ and an absolute accuracy of $25 \mathrm{~cm}$ was available for the test sites [29]. Table 1 summarizes the characteristics of the individual sample plots. The descriptive statistics of the field trees are summarized in Table 5.

Table 5. Statistical summary of field tree variables in Test Site I.

\begin{tabular}{ccccc}
\hline Time of acquisition & \multicolumn{2}{c}{ May 2006 } & \multicolumn{2}{c}{ May 2007 } \\
\hline & Tree height $(\mathrm{m})$ & DBH $(\mathrm{cm})$ & Tree height $(\mathrm{m})$ & DBH $(\mathrm{cm})$ \\
Min & 5.10 & 7 & 5.10 & 7 \\
Max & 50.60 & 113 & 50.60 & 113 \\
Mean & 25.42 & 31.90 & 25.29 & 31.70 \\
Standard deviation & 10.70 & 17.90 & 10.68 & 17.60 \\
\hline
\end{tabular}




\subsubsection{Test Site II}

We applied the strategy to the sample plots acquired under leaf-off condition and validated the results using the method of single-tree based evaluation. Reference data for all trees with DBH larger than $15 \mathrm{~cm}$ were collected for 62 Norway spruces (Picea abies), one European beeches (Fagus sylvatica), 24 European ash (Fraxinus excelsior), 32 Black Alder (Alnus glutinosa), four Sycamore maples (Acer pseudoplatanus), and one lime trees (Tilia Europaea). Tree parameters including the $\mathrm{DBH}$, total tree height, stem position and tree species were measured and determined by GPS, tachometry and the "Vertex III hypsometer" system. Additionally, a DTM with a grid size of $1 \mathrm{~m}$ and an absolute accuracy of $25 \mathrm{~cm}$ was also available. The descriptive statistics of the field trees are summarized in Table 6.

Table 6. Statistical summary of field tree variables in Test Site II.

\begin{tabular}{ccc}
\hline Time of acquisition & \multicolumn{2}{c}{ March 2011 } \\
\hline Min & Tree height $(\mathrm{m})$ & DBH $(\mathrm{cm})$ \\
Max & 1.0 & 2.0 \\
Mean & 44.0 & 50.0 \\
Standard deviation & 21.2 & 22.8 \\
\end{tabular}

\subsubsection{Evaluation}

The tree detection evaluation is performed based on the single-tree level by finding matched tree segments in the reference. The tree detection results are evaluated by comparison with reference data using two criteria: (i) the distance from detected trees to reference should be smaller than $60 \%$ of the mean tree spacing of the plot; (ii) the height difference between detected and reference trees should be

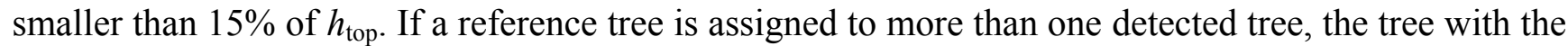
minimum distance to the reference is selected. Detected trees that are linked to one reference tree are so-called "true positives" and detected trees without any link to a tree position are treated as "false positives". We used "completeness and correctness" measures to characterize the performance of the individual tree detection, which are defined in Equations (4) and (5), respectively.

$$
\begin{gathered}
\text { Completeness }=\frac{\text { Number }_{\text {true positives }}}{\text { Number }_{\text {reference tree }}} \\
\text { Correctness }=\frac{\text { Number }_{\text {true positives }}}{\text { Number }_{\text {detected tree }}}
\end{gathered}
$$

\section{Results and Discussion}

\subsection{Results}

By applying the procedure for $3 \mathrm{D}$ single tree detection to the test plots in a batch procedure, the experimental results describing the impact of each selected control parameter on the performance of 
tree detection are illustrated in Figures 3-11. Since the sensitivity analysis is performed based on varying one control parameter at each time, those parameters which did not vary during the sensitivity analysis should be assigned with constant values: $N C u t_{\text {Thre }}=0.16, V_{\text {size }}=0.5, \lambda=1.5$.

\subsubsection{Test Site I}

Figures 3-5 show the results for the dataset of Test Site I acquired in 2006 under leaf-off condition, while Figures 6-8 show the results for Test Site I acquired in 2007 under leaf-on condition.

Figure 3. Sensitivity analysis with respect to the parameter $N C u t_{\text {Thres }}$ for the dataset of Test Site I acquired under leaf-off condition; (a) Completeness; (b) Correctness; colored lines indicate the forest layers.

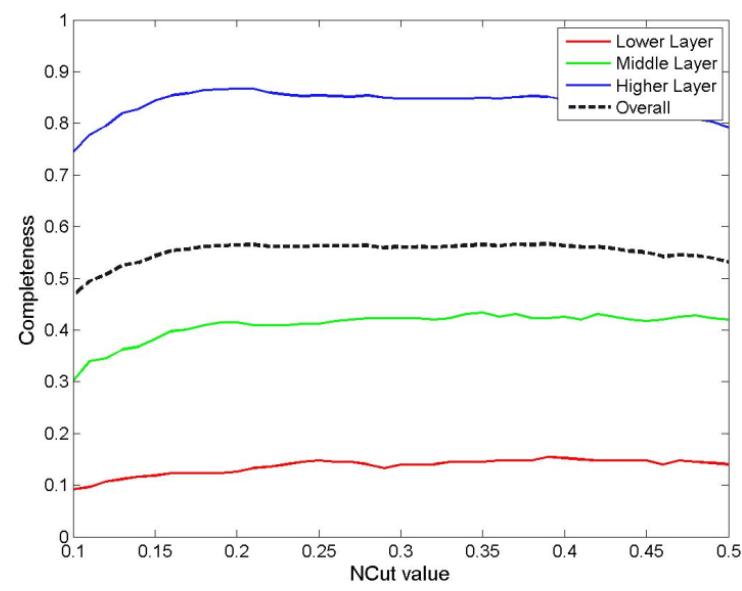

(a)

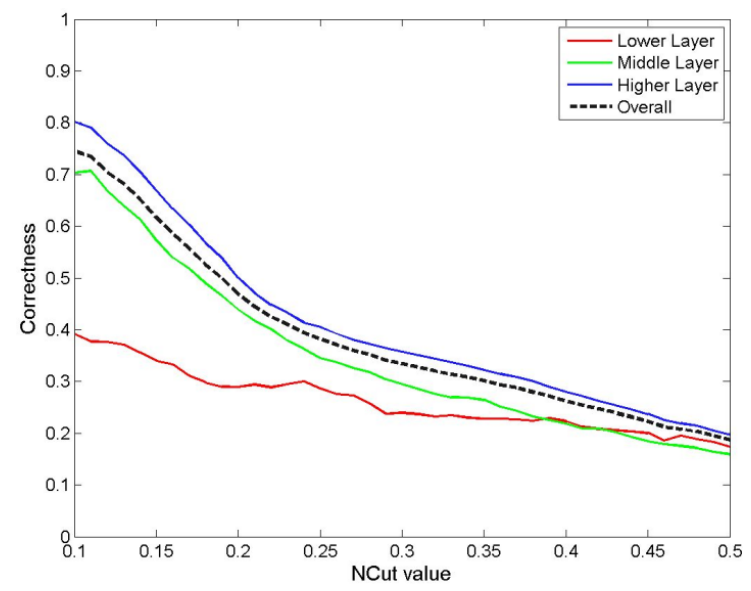

(b)

Figure 4. Sensitivity analysis with respect to the parameter $V_{\text {Size }}$ for the dataset of Test Site I acquired under leaf-off condition; (a) Completeness; (b) Correctness; colored lines indicate the forest layers.

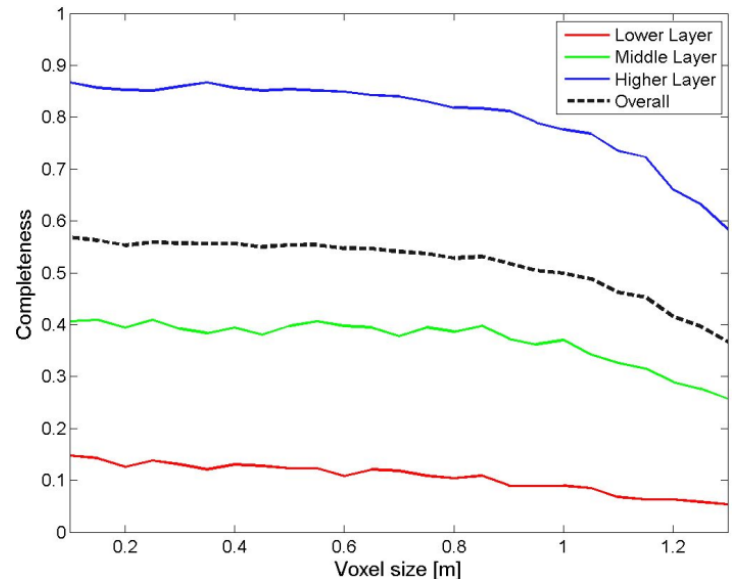

(a)

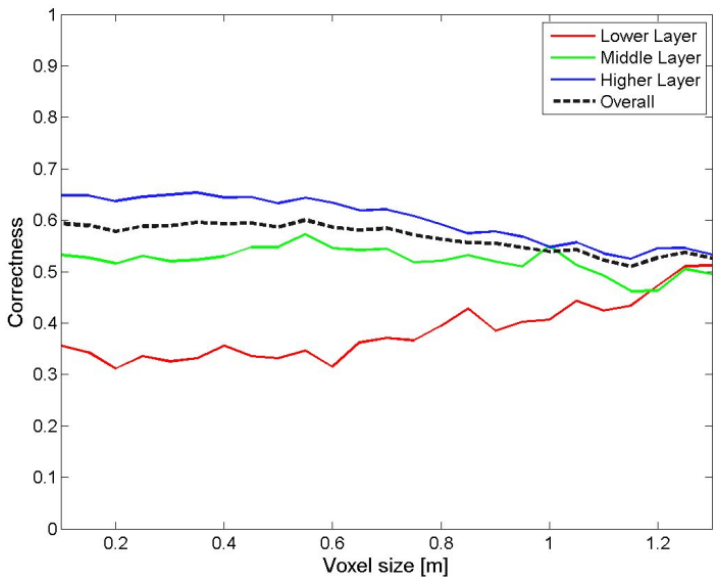

(b) 
Figure 5. Sensitivity analysis with respect to the parameter $\lambda$ for the dataset of Test Site I acquired under leaf-off condition; (a) Completeness; (b) Correctness; colored lines indicate the forest layers.

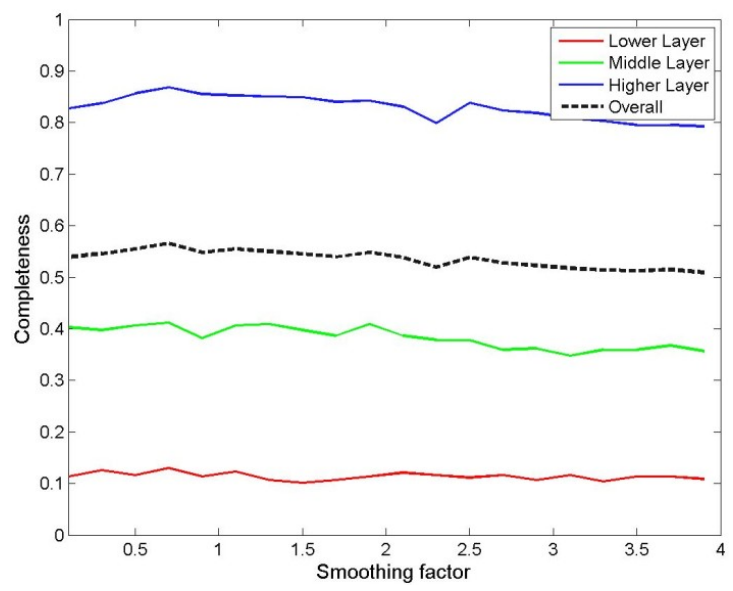

(a)

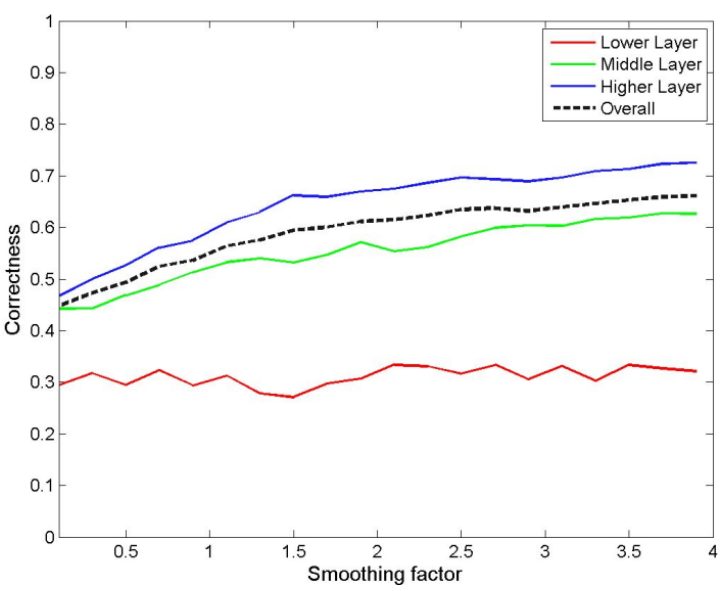

(b)

Figure 6. Sensitivity analysis with respect to the parameter $N C u t_{\text {Thres }}$ for the dataset of Test Site I acquired under leaf-on condition; (a) Completeness; (b) Correctness; colored lines indicate the forest layers.

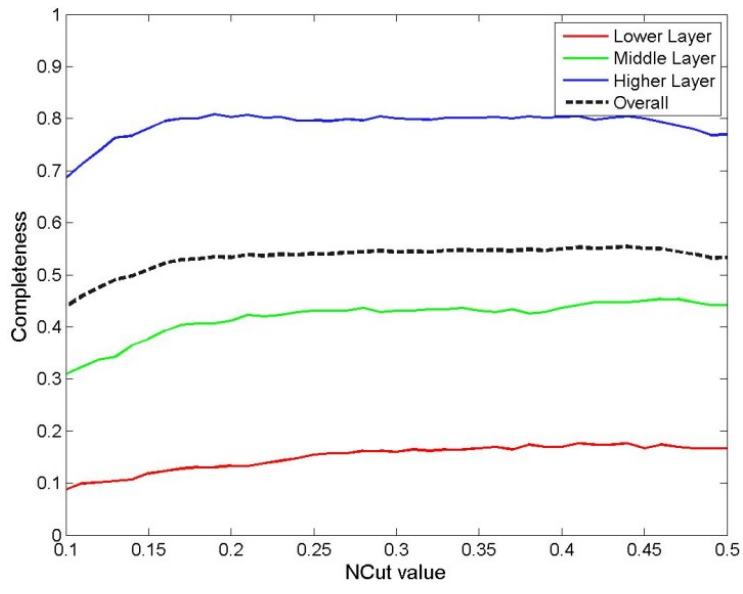

(a)

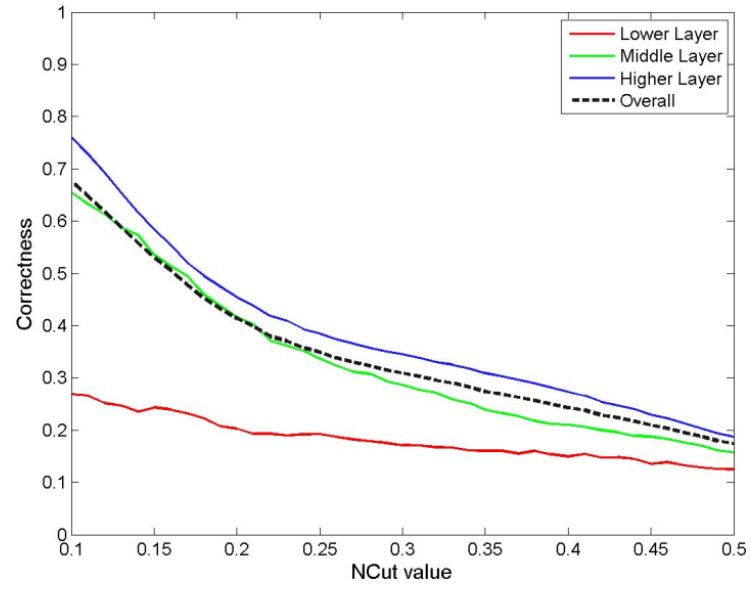

(b) 
Figure 7. Sensitivity analysis with respect to the parameter $V_{\text {Size }}$ for the dataset of Test Site I acquired under leaf-on condition; (a) Completeness; (b) Correctness; colored lines indicate the forest layers.

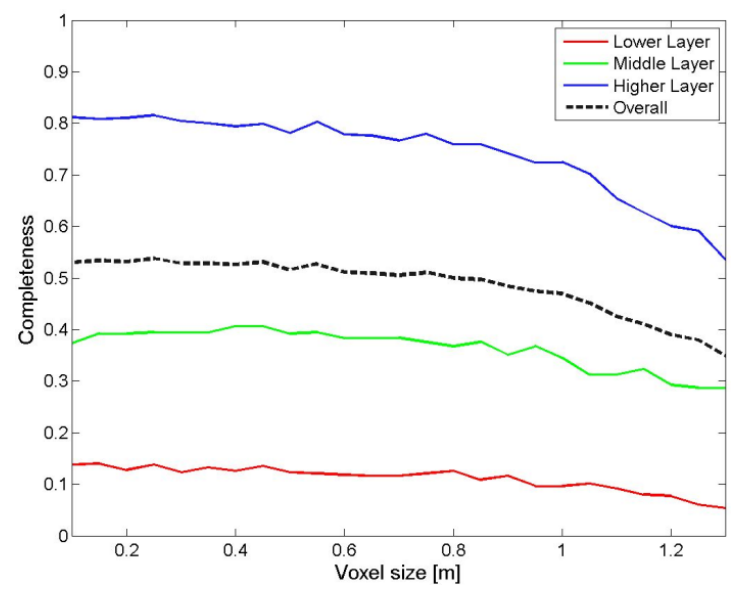

(a)

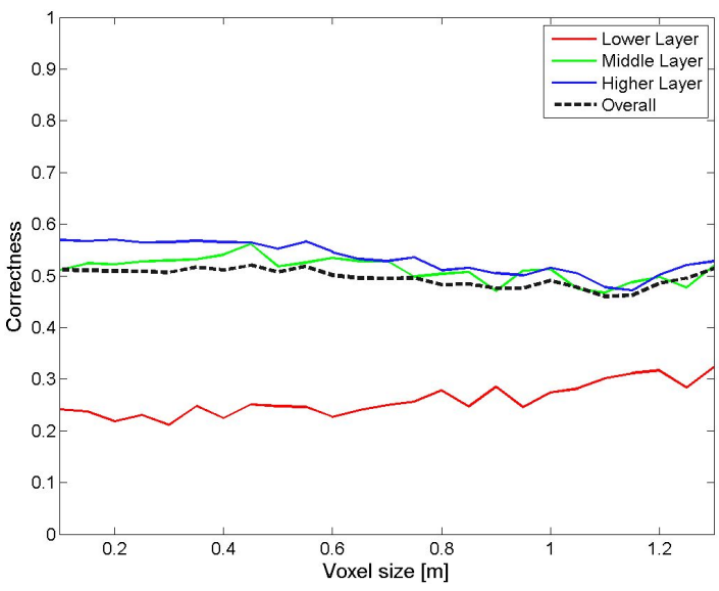

(b)

Figure 8. Sensitivity analysis with respect to the parameter $\lambda$ for the dataset of Test Site I acquired under leaf-on condition, (a) Completeness; (b) Correctness; colored lines indicate the forest layers.

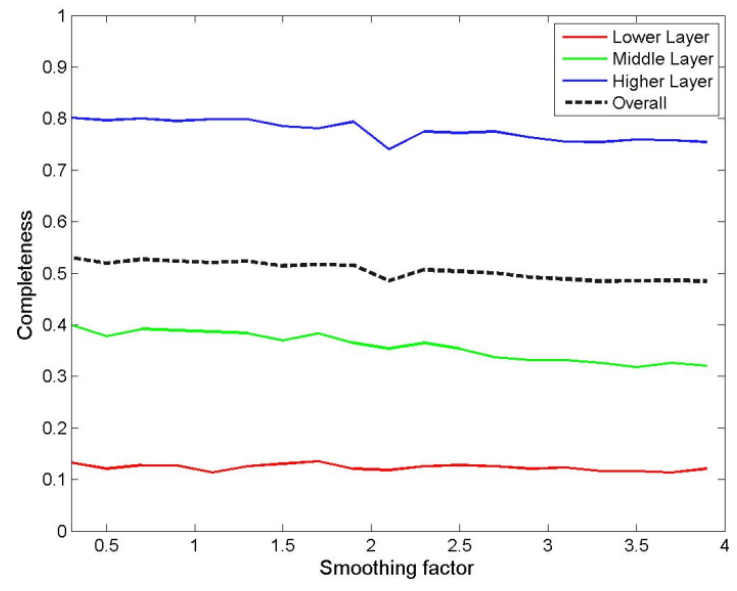

(a)

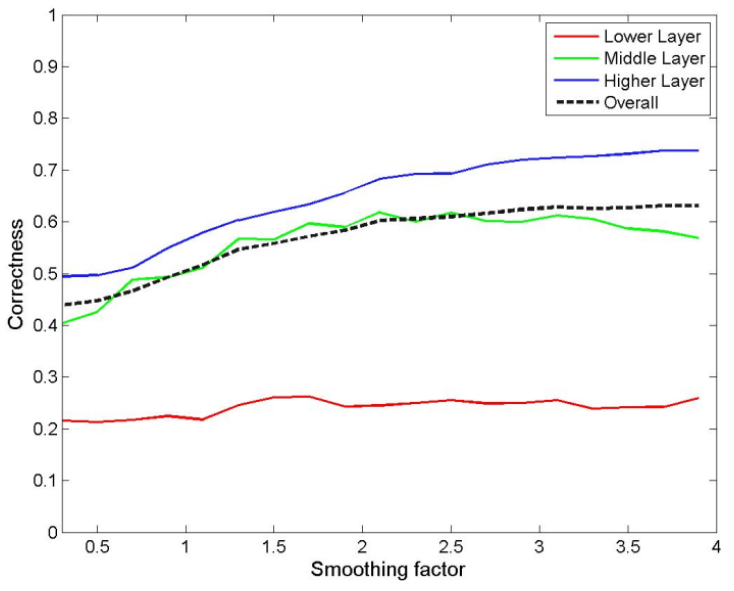

(b) 


\subsubsection{Test site II}

Figures 9-11 show the results for the dataset of Test Site II.

Figure 9. Sensitivity analysis with respect to the parameter $N C u t_{\text {Thres }}$ for the dataset of Test Site II acquired under leaf-off condition; (a) Completeness; (b) Correctness; colored lines indicate different point densities.

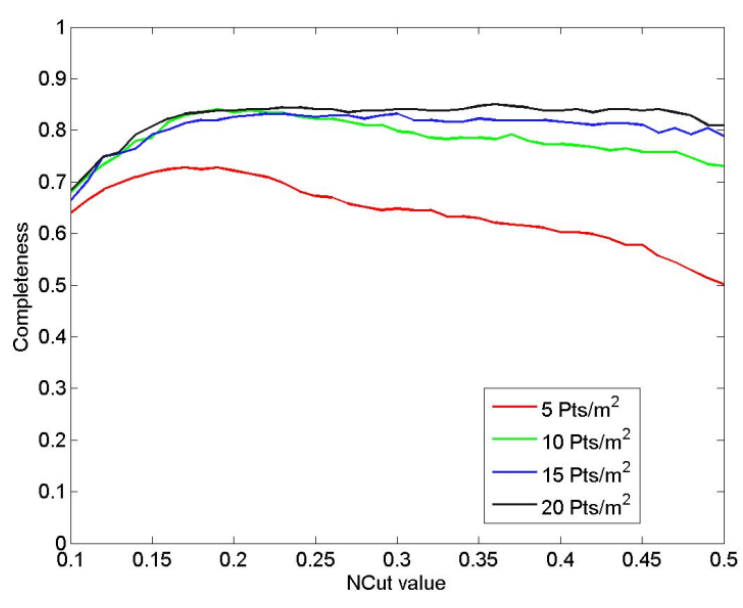

(a)

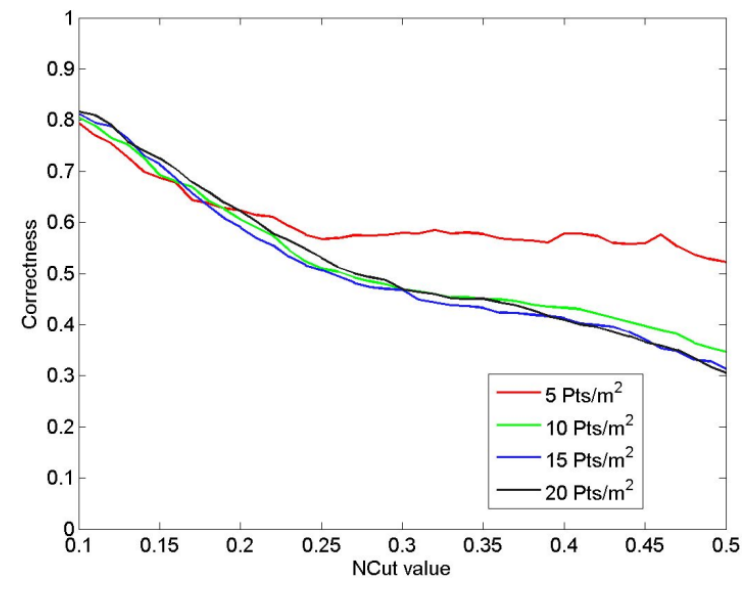

(b)

Figure 10. Sensitivity analysis with respect to the parameter $V_{\text {Size }}$ for the dataset of Test Site II acquired under leaf-off condition; (a) Completeness; (b) Correctness; colored lines indicate different point densities.

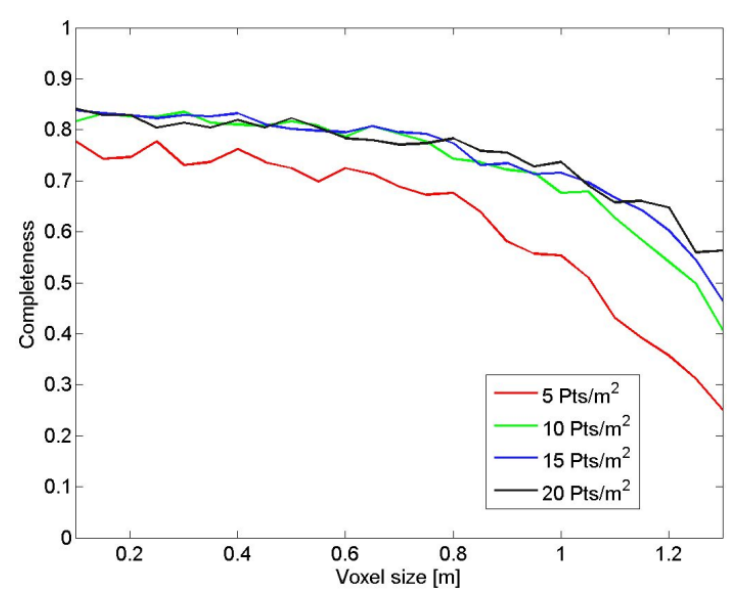

(a)

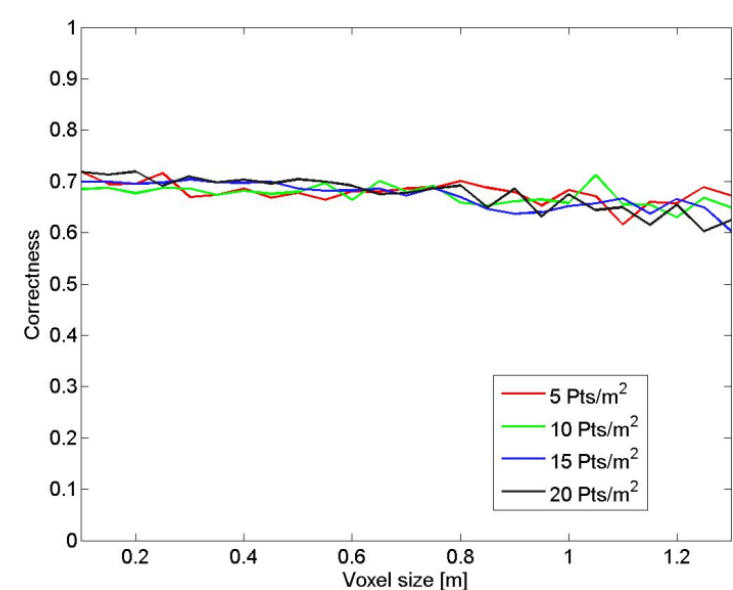

(b) 
Figure 11. Sensitivity analysis with respect to the parameter $\lambda$ for the dataset of Test Site II acquired under leaf-off condition; (a) Completeness; (b) Correctness; colored lines indicate different point densities.

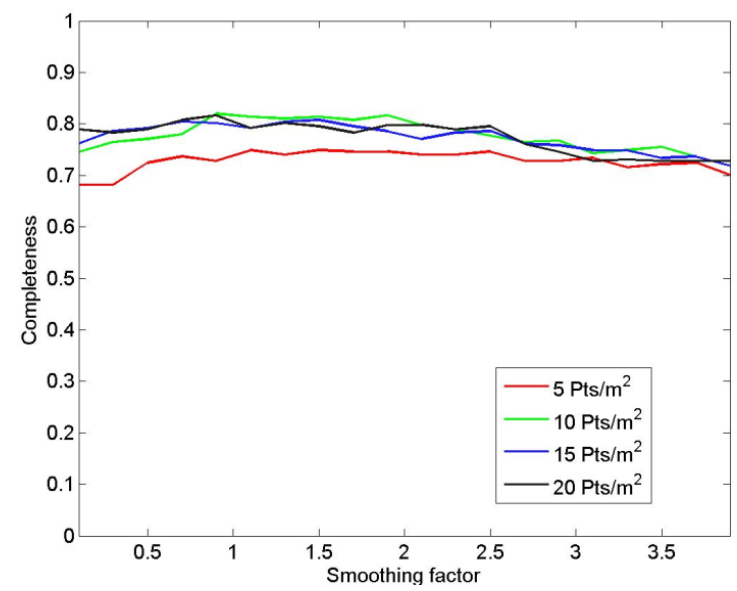

(a)

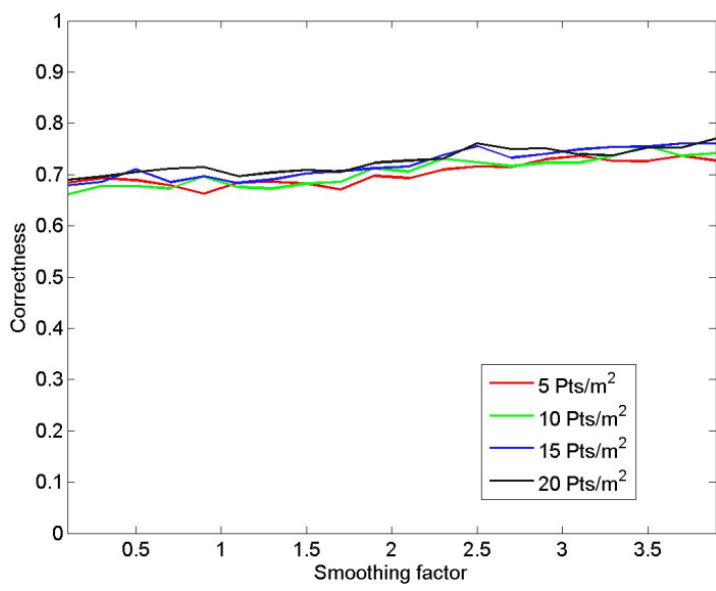

(b)

\subsection{Discussion}

\subsubsection{Test Site I vs. Test Site II}

The two test sites are characterized by different forest structures and stand properties, although they are located in the same geographical zone. Test Site I is characterized by a multi-story forest area with uneven-aged stands. Test Site II features a more homogenous forest area with a dominant upper layer and monocultural characteristics. By comparing the variation curves of the two data sites in Figures 3-11 in response to the control parameters shows that the trend of the uncertainty of the tree detection performance with respect to the different algorithm parameters seems to remain stable. It verifies the important expectation that the proposed 3D segmentation method can deal with forest areas of different structural properties in a robust manner. The quantitative results for tree detection certainly become degraded if test areas have more challenging forest structures such as high stem density, mixed stand and multi-layered trees. Consequently, one can generally observe that the detection rate in Test Site II is about $20 \%$ higher than that in Test Site I, while the test site I features a $10 \%$ larger false positive rate. Moreover, according to Figures 5, 8, and 11 the smoothing factor $\lambda$ is likely to have more distinct effects on the correctness of the tree detection results for Test Site I. The false alarm rate in Test Site I was reduced by at least $15 \%$ whereas that in Test Site II was reduced only slightly. This suggests that it is beneficial to smooth the CHM of multi-layered forest areas prior to 3D segmentation in order to eliminate redundant local maxima contributed by many small trees, since the completeness of tree detection for both test sites remained stable when the smoothing factor $\lambda$ increased from one to four. 


\subsubsection{Foliage Condition}

Since LiDAR datasets were only acquired under leaf-on condition at Test Site II, the sensitivity analysis with respect to the influence of the foliage condition is limited to Test site I. Figures 3-8 show the sensitivity analysis results of Test Site I under two foliage conditions-leaf-on and leaf-off, respectively. Generally, the impact of the varied control parameters on the tree detection performance is not significantly biased towards the foliage condition as observed in figures for the different parameters. For completeness, overall results for tree detection neither differ much in the absolute value nor in the trend of the curve changing with an increasing parameter. Neither can one find any distinct difference among the forest height layers between leaf-on and off foliage conditions. Only trees within the upper height layer are relatively more affected, which indicates a slight improvement concerning the detection rate in the case of leaf-off conditions. It matched the assumption that tall trees in the overstory should be better detected in the leaf-off case due to the weaker saturation effect of tree crowns (as top foliage of the tree crown reflects most of the signal back in the leaf-on case) and better reflection of inner tree structure caused by higher canopy transmittance against laser pulse. Nevertheless, the dependency relationship of the performance indices on the parameters is still maintained constant, no matter which forest height layer is considered. When one takes a look at the performance aspect concerning the correctness, a lower forest height layer seems to be the component of most concern. The leaf-on case decreased the correctness of tree detection in the lower forest layer by ca. $10 \%$, while sensitivity curves of the control parameters tended to become steadier at all the forest height layers. Especially for the voxel size parameter the correctness of tree detection in the lower height layer did not result in on any significant improvement as in the leaf-off case for voxel sizes larger than $1.2 \mathrm{~m}$. Incomplete sampling of small trees in the lower layer under leaf-on condition leads to the information loss in the voxel unit which was even more extensive. Additionally, it is comprehensible to see that trees in the lower height layer are not sensible to the variation of the smoothing factor applied to CHM, since most of the trees in that layer are not represented in the CHM, which are of course not affected by the smoothing operation.

\subsubsection{Point Density}

The experimental results of the sensitivity analysis for both Test Site I and Test Site II with four-fold point density demonstrate that the threshold $N C u t_{\text {Thres }}$ plays a more significant role in the determination of overall performance of $3 \mathrm{D}$ tree detection from airborne LiDAR point clouds. The optimal value for $N C u t_{\text {Thres }}$ should be selected between 0.16 and 0.23 in order to achieve the trade-off between the completeness and correctness. Moreover, all other control parameters except $N C u t_{\text {Thres }}$ are much less sensitive with regard to the correctness and completeness for point densities larger than $5 \mathrm{pts} / \mathrm{m}^{2}$.

Larger $N C u t_{\text {Thres }}$ values lead to more tree segments with a higher completeness but lower correctness (over-segmentation), since the $N C u t_{\text {Thres }}$ value defines the threshold for evaluating the dissimilarity measure between sub-graphs. Lower point density datasets including one-fold $\left(5 \mathrm{pts} / \mathrm{m}^{2}\right)$ or two-fold $\left(10 \mathrm{pts} / \mathrm{m}^{2}\right)$ datasets even produce a descending completeness when $N C u t_{\text {Thres }}$ is larger than 0.4 . Such low point density datasets reach their over-segmentation limit and will produce no more 
segments on the basis of bipartition cuts. However, the performances of 3D tree segmentation for the data sets of four different point densities are almost identical if a $N C u t_{\text {Thres }}$ value smaller than 0.25 is selected. The performance curves resulting from varying the smoothing factor $\lambda$ indicate it to have only negligible significance regardless of the point density of the LiDAR data. Only the correctness appears to slightly increase. In response, the evolution of performance curves with respect to $\lambda$ (Figure 11) looks like a stochastic fluctuation around a constant value. The results from the variation of the voxel size $V_{\text {size }}$ show that there is a slightly consistent impact of $V_{\text {size }}$ on the performance of the $3 \mathrm{D}$ tree detection leading to a degraded completeness when $V_{\text {size }}$ increases, since the progress of correctness curves follows a fluctuant distribution around the same value. Datasets with lower point density under $10 \mathrm{pts} / \mathrm{m}^{2}$ are especially sensible to the large voxel size whereby the completeness decreases dramatically if $V_{\text {size }}$ exceeds $0.6 \mathrm{~m}$.

An interesting finding is that a higher point density $\left(>10 \mathrm{pts} / \mathrm{m}^{2}\right)$ cannot necessarily guarantee better performance or stable results for tree detection given the varied voxel size. Moreover, if the voxel size increases to more than $0.8 \mathrm{~m}$ an under-segmentation happens to the one/two-fold point density datasets. This could be due to the fact that the large voxel size reduces the dimension of the adjacency graph and small trees are unified into one graph node. Generally, the point density of LiDAR data seems to not play as significant a role as expected. In this case, it is difficult to state that the performance of the tree detection algorithm really benefits from high point density, at least for those datasets with a point density larger than $10 \mathrm{pts} / \mathrm{m}^{2}$. High point density $\left(>10 \mathrm{pts} / \mathrm{m}^{2}\right)$ seems not to contribute to any significant improvement to the tree detection results. A similar saturation effect in point density for tree detection from LiDAR data was also observed and reported in the study [20], in which the point density increased from two points to eight points per $\mathrm{m}^{2}$ and had a marginal improvement in crown delineation accuracy.

The study performed in [30] confirmed that the accuracy of predicted metrics at the plot level such as tree height $\mathrm{DBH}$ and basal area was also invariant to changes in pulse density at moderate to high densities no matter what kind of prediction models were used. Coverage metric including canopy cover and tree density are more sensitive to changes in point density but still not significant. They suggest that low-density LiDAR data may be capable of estimating typical forest structure metrics reliably in some situations. This study enlightens the user to achieve the trade-off between data acquisition cost and accuracy of tree detection in forest inventory products where time expenses for flight and post-processing can be simultaneously minimized at no extra cost of data quality. The study indicates that the point density may not be the major factor, which should be pursued in the data preparation for LiDAR forestry remote sensing. It may be a result of the fact that the vertical resolving ability of LiDAR towards forestry tasks is more relevant to the penetrability of laser pulses against tree crown rather than the point spacing.

\section{Conclusions}

It is necessary to characterize and even quantify the influence of uncertainty associated with various control parameters for single tree detection based on 3D segmentation of LiDAR point clouds, since the performance of the forest characterization based on ITD strategy is directly related to the accuracy of tree detection results. The parameter settings of the tree detection method needs to be examined and 
optimized towards different data acquisition conditions and forest stand properties, such as point density, flight altitude, forest type and tree growth phase. The experimental results of this work show that the threshold for normalized cut value $N C u t_{\text {Thres }}$ is the most important and influential control parameter in the entire $3 \mathrm{D}$ segmentation algorithm. $N C u t_{\text {Thres }}$ is also the most sensible parameter to the performance of tree detection and needs to be carefully adjusted. According to the sensitivity analysis performed here, the developed strategy for tree detection remains steady with respect to dealing with various forest structures, even though multi-layered mixed forest stands may show degraded results due to the existence of an understory. Additionally, foliage condition is more likely to have a distinct impact on the correctness of tree detection in forest understory, especially with regard to the selection of the voxel size. In particular, this means that it is feasible to enlarge $V_{\text {size }}$ to alleviate the oversegmentation effect for understory trees in leaf-on case. Finally, high point density of LiDAR data leads to high data acquisition cost and time expenses (especially for the waveform LiDAR extra time expenses for waveform decomposition are needed), but not performance improvement in proportion. The value of $10 \mathrm{pts} / \mathrm{m}^{2}$ seems to be a good trade-off between the cost and the quality of results.

Future work could be focused on the simultaneous optimization of multiple control parameters and the extension to the sensitivity analysis for tree species classification and forest parameter estimation. Furthermore, the sensitivity analysis on LiDAR data will enable us to identify the most influential structural parameters of forests for tree detection results, such as stem density and tree height distribution.

\section{Acknowledgments}

The authors want to thank Mr. Ing. Hermann Novak from Forest Mapping Management GmbH in Salzburg, Austria for providing us the experimental and field reference data of Test Site II.

\section{Author Contributions}

Wei Yao wrote the manuscript and was responsible for the research design and analysis. Jan Krull was responsible for experimental implementation as well as data analysis. Peter Krzystek supported the analysis and technical implementation. Marco Heurich provided the reference data from field works.

\section{Conflicts of Interest}

The authors declare no conflict of interest.

\section{References}

1 Yao, W.; Stilla, U. Mutual enhancement of weak laser pulses for point cloud enrichment based on full-waveform analysis. Geosci. Remote Sens. 2010, 48, 3571-3579.

2 Vaughn, N.R.; Moskal, L.M.; Turnblom, E.C. Tree species detection accuracies using discrete point LiDAR and airborne waveform LiDAR. Remote Sens. 2012, 4, 377-403. 
3 Yao, W.; Krzystek, P.; Heurich, M. Tree species classification and estimation of stem volume and DBH based on single tree extraction by exploiting airborne full-waveform LiDAR data. Remote Sens. Environ. 2012, 123, 368-380.

4 Stilla, U.; Yao, W.; Jutzi, B. Detection of weak laser pulses by full waveform stacking. In PIA07 Photogrammetric Image Analysis 2007; International Archives of Photogrammetry, Remote Sensing, and Spatial Information Sciences; Stilla, U., Ed.; Volume 36(3/W49A), pp. 25-30.

5 Reitberger, J.; Krzystek, P.; Stilla, U. Analysis of full waveform LiDAR data for the classification of deciduous and coniferous trees. Int. J. Remote Sens. 2008, 29, 1407-1431.

6 Næsset, E. Predicting forest stand characteristics with airborne scanning laser using a practical two-stage procedure and field data. Remote Sens. Environ. 2002, 80, 88-99.

7 White, J.C.; Wulder, M.A.; Vastaranta, M.; Coops, N.C.; Pitt, D.; Woods, M. The utility of image-based point clouds for forest inventory: A comparison with airborne laser scanning. Forests 2013, 4, 518-536.

8 He, Q.; Chen, E.; An, R.; Li, Y. Above-ground biomass and biomass components estimation using LiDAR data in a coniferous forest. Forests 2013, 4, 984-1002.

9 Andersen, H.E.; McGaughey, R.J.; Reutebuch, S.E. Estimating forest canopy fuel parameters using LiDAR data. Remote Sens. Environ. 2005, 94, 441-449.

10 Nelson, R.; Krabill, W.; Tonelli, J. Estimating forest biomass and volume using airborne laser data. Remote Sens. Environ. 1988, 24, 247-267.

11 Heurich, M.; F. Thoma. Estimation of forestry stand parameters using laser scanning data in temperate, structurally rich natural beech (Fagus sylvatica) and spruce (Picea abies) forests. Forestry 2008, 81, 645-661.

12 Kantola, T.; Vastaranta, M.; Lyytikäinen-Saarenmaa, P.; Holopainen, M.; Kankare, V.; Talvitie, M.; Hyyppä, J. Classification of needle loss of individual Scots pine trees by means of airborne laser scanning. Forests 2013, 4, 386-403.

13 Ene, L.; Næsset, E.; Gobakken, T. Single tree detection in heterogeneous boreal forests using airborne laser scanning and area-based stem number estimates. Int. J. Remote Sens. 2012, $33,5171-5193$.

14 Persson, A.; Holmgren, J.; Soderman, U. Detecting and measuring individual trees using an airborne laser scanner. Photogramm. Eng. Remote Sens. 2002, 68, 925-932.

15 Yu, X.; Hyyppä, J.; Vastaranta, M.; Holopainen, M.; Viitala, R. Predicting individual tree attributes from airborne laser point clouds based on the random forests technique. ISPRS J. Photogramm. Remote Sens. 2011, 64, 28-37.

16 Reitberger, J.; Schnörr, C.; Krzystek, P.; Stilla, U. 3D segmentation of single trees exploiting full waveform LiDAR data. ISPRS J. Photogramm. Remote Sens. 2009, 64, 561-574.

17 Vauhkonen, J.; Ene, L.; Gupta, S.; Heinzel, J.; Holmgren, J.; Pitkänen, J.; Solberg, S.; Wang, Y.; Weinacker, H.; Hauglin, K.M.; Lien, V.; Packalén, P.; Gobakken, T.; Koch, B.; Næsset, E.; Tokola, T.; Maltamo, M. Comparative testing of single tree detection algorithms under different types of forest. Forestry 2012, 3, 27-40.

18 Abed, F.M.; Mills, J.P.; Miller, P.E. Echo amplitude normalization of full-waveform airborne laser scanning data based on robust incidence angle estimation. IEEE Trans. Geosci. Remote Sens. 2012, 50, 2910-2918. 
19 Korpela, I.; Ørka, H.O.; Maltamo, M.; Tokola, T.; Hyyppä, J. Tree species classification using airborne LiDAR-effects of stand and tree parameters, downsizing of training set, intensity normalization, and sensor type. Silva Fenn. 2010, 44, 319-339.

20 Kaartinen, H.; Hyyppä, J.; Yu, X.; Vastaranta, M.; Hyyppä, H.; Kukko, A.; Holopainen, M.; Heipke, C.; Hirschmugl, M.; Morsdorf, F.; Næsset, E.; Pitkänen, J.; Popescu, S.; Solberg, S.; Wolf, B.M.; Wu, J.C. An international comparison of individual tree detection and extraction using airborne laser scanning. Remote Sens. 2012, 4, 950-974.

21 Gonzalez, P.; Asner, G.P.; Battles, J.; Lefsky, M.A.; Waring, K.M.; Palace, M. Forest carbon densities and uncertainties from LiDAR, quickbird, and field measurements in California. Remote Sens. Environ. 2011, 114, 1561-1575.

22 Lu, D.; Chen, Q.; Wang, G.; Moran, E.; Batistella, M.; Zhang, M.; Laurin, G.V.; Saah, D. Aboveground forest biomass estimation with landsat and LiDAR data and uncertainty analysis of the estimates. Int. J. For. Res. 2012, doi:10.1155/2012/436537.

23 Monnet, J.M.; Merminy, E.; Chanussotz, J.; Berger, F. Tree top detection using local maxima filtering: a parameter sensitivity analysis. In Proceedings of the 10th International Conference on LiDAR Applications for Assessing Forest Ecosystems (Silvilaser 2010), Freiburg, Germany, September 2010; pp. 14-17.

24 Palleja, T.; Tresanchez, M.; Teixido, M.; Sanz, R.; Rosell, J.R.; Palacin, J. Sensitivity of tree volume measurement to trajectory errors from a terrestrial LiDAR scanner. Agric. For. Meteorol. 2010, 150, 1420-1427.

25 Mutlu, M.; Popescu, S.C.; Zhao, K. Sensitivity analysis of fire behavior modeling with LiDAR derived surface fuel maps. For. Ecol. Manag. 2008, 256, 289-294.

26 D’Errico, J. 2006, Surface fitting using gridfit. Available online: http://www.mathworks.com/ matlabcentral/fileexchange (accessed on 1 October 2009).

27 Shi, J.; Malik, J. Normalized cuts and image segmentation. Pattern Anal. Mach. Intell. 2000, 22, 888-905.

28 Reitberger, J. 3D-Segmentierung von Einzelbäumen und Baumartenklassifikation aus Daten flugzeuggetragener Full Waveform Laserscanner. Ph.D. Thesis, Technische Universitaet Muenchen, München, Germany, 2010.

29 Heurich, M. Automatic recognition and measurement of single trees based on data from airborne laser scanning over the richly structured natural forests of the Bavarian Forest National Park. For. Ecol. Manag. 2008, 255, 2416-2433.

30 Jakubowski, M.K.; Guo, Q.; Kelly, M. Tradeoffs between LiDAR pulse density and forest measurement accuracy. Remote Sens. Environ. 2013, 130, 245-253.

(C) 2014 by the authors; licensee MDPI, Basel, Switzerland. This article is an open access article distributed under the terms and conditions of the Creative Commons Attribution license (http://creativecommons.org/licenses/by/3.0/). 